\title{
Cosmic ray induced explosive chemical desorption in dense clouds
}

\author{
C. J. Shen, J. M. Greenberg ${ }^{\star}$, W. A. Schutte, and E. F. van Dishoeck
}

Leiden Observatory, Leiden University, PO Box 9513, 2300 RA Leiden, The Netherlands

Received 3 January 2003 / Accepted 29 October 2003

\begin{abstract}
The desorption due to the energy release of free radicals in the ice mantles of a dust grain is investigated theoretically by calculating the ultraviolet radiation field inside the cloud, the free radical accumulation, the cosmic-ray heating of the grain and then the desorption in this situation starting from the cosmic-ray energy spectra. This model can reproduce the observations of the CO gas abundances and level of depletion in dark clouds such as L977 and IC 5146 with a combination of input parameters which are either constrained by independent observations or have been derived independently from laboratory experiments. We investigate other desorption mechanisms and conclude that they cannot explain the observations. The model also shows that the energy input by the cosmic-ray induced ultraviolet field is almost one order of magnitude larger than the direct energy input by cosmic-ray particles. This strengthens the conclusion that desorption due to the energy release by ultraviolet photon produced radicals dominates over direct cosmic-ray desorption.
\end{abstract}

Key words. ISM: dust, extinction - ISM: cosmic rays - ISM: clouds - ISM: individual: objects: L977, IC 5146

\section{Introduction}

Inside cold dense interstellar clouds, all heavy molecules will stick to the grain, forming an ice mantle upon collision with an efficiency close to unity. Chemical models of these regions (e.g. Willacy \& Millar 1998) show that all heavy molecules are removed from the gas phase within $\sim 10^{9} / n_{\mathrm{H}}$ years, where $n_{\mathrm{H}}$ is the total hydrogen number density. For a typical density of $10^{4} \mathrm{~cm}^{-3}$, this time scale is much shorter than the estimated age of such regions. Therefore, all molecules other than $\mathrm{H}_{2}$ should not be available in the gas phase in these clouds. However, observations of these regions show that both solid phase and gas phase molecules such as $\mathrm{CO}$ and $\mathrm{N}_{2} \mathrm{H}^{+}$exist (Alves et al. 1999; Bergin et al. 2001). Thus some desorption mechanisms are needed to keep part of the heavy molecules in the gas phase.

Clarifying the desorption mechanisms is important in understanding the physical and chemical evolution of the interstellar clouds for a number of reasons. First, they control the allocation of molecules between gas and solid phase, which affects the gas phase and surface reactions as well as the dust properties. Second, the gas composition of dense clouds is strongly influenced by the kind of molecules that sublimate from the grains. The molecules that stick on the grain surface may undergo surface reactions with other species; and the molecules that sublimate from the surface may well be different from those that stick on the surface. Finally, the desorption mechanism will determine the history and composition of the ice mantles.

Send offprint requests to: E. F. van Dishoeck, e-mail: ewine@strw.leidenuniv.nl

* Deceased on November 29, 2001.
Several mechanisms have been postulated to prevent the problem of complete freeze-out of heavy molecules in dense clouds. The first is photon-desorption, which is important at the cloud edge, where a lot of ultraviolet (UV) photons are present, but it is negligible in the inner part due to extinction (Tielens \& Hagen 1982; d'Hendecourt et al. 1985). Although the cosmic-ray induced UV field (Prasad \& Tarafdar 1983) may play a role, it is far from sufficient to maintain the observed gas phase abundances (Willacy \& Millar 1998). The second mechanism is the cosmic-ray whole grain heating, which is also not efficient enough by itself (Willacy \& Millar 1998). Léger et al. (1985) suggested that cosmic-ray spot heating could be very important to maintain gas phase molecules in dense regions. However, as will be discussed in Sect. 7, new estimates indicate that this mechanism is also quite insufficient. Duley \& Williams (1993) proposed that the chemical desorption of weakly bound molecules such as $\mathrm{CO}$ may occur in the vicinity of $\mathrm{H}_{2}$-forming sites on dust grains because a small release of $\mathrm{H}_{2}$ formation energy may be sufficient to heat dust locally. However, this desorption only works for grains as small as $20 \AA$ (Takahashi \& Williams 2000), which is not the case in such dense regions. A desorption mechanism involving the release or even explosive release of the chemical energy stored in the ice mantles of the dust grain as free radicals by previous UV photolysis was proposed by Greenberg and co-workers on the basis of theoretical arguments as well as laboratory simulation (Greenberg \& Yencha 1973; d'Hendecourt et al. 1982; Schutte \& Greenberg 1991). In this paper we will further explore and compare these various mechanisms of desorption. 
Recently, Lada et al. (1994) developed a powerful technique for mapping the large-scale distribution of dust using multi-wavelength near-infrared imaging. By measuring the near-infrared color excess of stars behind a cloud, the lineof-sight dust extinction (and hence, the total column density) can be determined directly. The technique allows measurements over a large range of scales and extinction. Using this method, the correlation between $\mathrm{CO}$ gas and dust in the dense cores L977 (Alves et al. 1999), IC 5146 (Kramer et al. 1999) and B68 (Bergin et al. 2002) has been obtained. We investigate here which desorption mechanisms can reproduce the observations.

In the very dense, shielded and quiescent regions of interstellar clouds, cosmic-ray particles are probably the only energetic source which can induce desorption. Therefore, the desorption mechanisms related to cosmic-ray particles, including the cosmic-ray whole grain heating (classical evaporation), the spot heating of large grains and the chemical energy release via radical reactions, must be the most important mechanisms for maintaining the observed gas-phase molecules such as CO. These mechanisms are investigated thoroughly from the theoretical point of view in this paper. To determine the desorption rates, several physical parameters, including the internal ambient and cosmic-ray induced UV fields, compositions of grain ice mantles, dust grain properties and the properties of the cosmic-ray induced heating events of a dust grain, need to be determined. In the dense region of a cloud, the UV photons and the grain heating are both directly related to the cosmicray spectra. The primary cosmic-ray spectrum is discussed in Sect. 2. In Sect. 3 we describe the dust model. The UV radiation field in dense clouds and the radical formation under this UV field are described in Sect. 4. The grain heating by cosmicray particles and energy inputs to ice mantles are investigated in Sect. 5. The radical formation in the grain ice mantles is covered in Sect. 6. In Sect. 7, the desorption rates of the various mechanisms are investigated. We compare the model results with the observations in Sect. 8, which is followed by a discussion.

\section{Primary cosmic ray spectra}

Galactic cosmic rays (GCRs) are high energy particles coming from outside the solar system, which can be measured directly. The GCR energy spectrum can be well represented by a powerlaw energy distribution for energies above $1 \mathrm{GeV}$ nucleon $^{-1}$ (Simpson 1983) but shows at low energies strong attenuation owing to the interaction between the solar wind and the cosmicray particles (Wiedenbeck \& Greiner 1980). For our purposes, it is important to constrain the cosmic ray energy spectra of protons and alpha particles which determine the cosmic ray ionization rate in the interstellar medium, and those of carbon, oxygen and iron (maybe magnesium and silicon as well) which can deposit enough energy on a dust grain upon a passing through to heat it to a higher temperature that leads to the desorption.

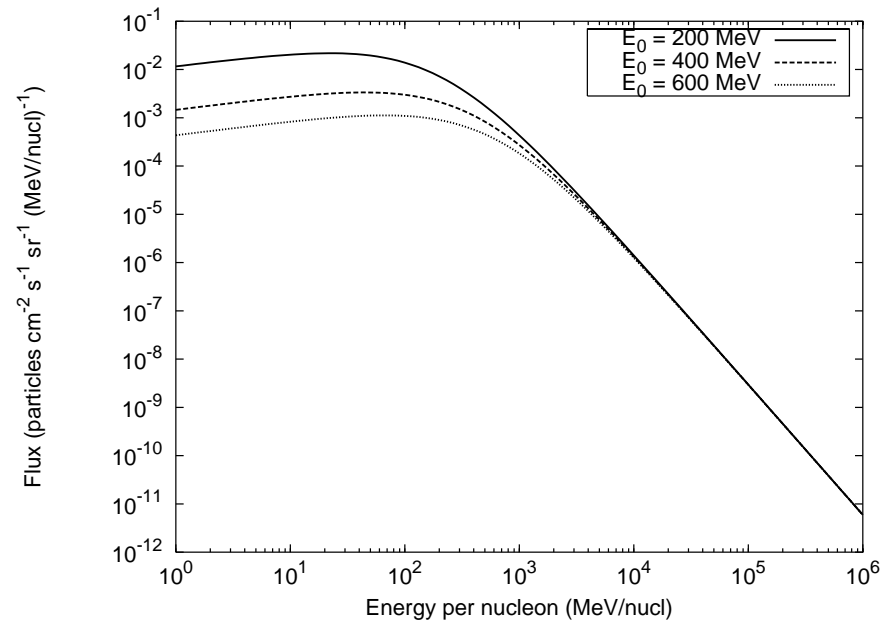

Fig. 1. Differential cosmic ray flux for protons in the energy range 1$10^{6} \mathrm{MeV}$ nucleon ${ }^{-1}$ in the interstellar medium. $E_{0}$ is a form parameter as in Eq. (1). Changing $E_{0}$ only affects the lower energy part of the spectrum. Lower $E_{0}$ means more low energy cosmic-ray particles.

Because of the strong modulation by solar winds, the lower energy tail of the galactic cosmic ray spectrum is very difficult to determine by direct measurements. However, the results from balloon and space-probe experiments, especially during the sunspot minimum period, have substantially improved our understanding of the cosmic ray spectrum below $1 \mathrm{GeV}$ per nucleon. If a solar modulation model is employed, the cosmic ray energy spectra can be inferred from the measurements near the earth. Based on the measurements of ${ }^{1} \mathrm{H},{ }^{2} \mathrm{H},{ }^{3} \mathrm{He}$ and ${ }^{4} \mathrm{He}$ made from balloon and Voyager experiments, Webber \& Yushak (1983) gave an approximated equation of primary cosmic ray spectra using the leaky box model for cosmic ray propagation and escape from the Galaxy:

$\frac{\mathrm{d} n}{\mathrm{~d} E}=\frac{C E^{0.3}}{\left(E+E_{0}\right)^{3}}$ particles $\mathrm{cm}^{-2} \mathrm{~s}^{-1} \mathrm{sr}^{-1}(\mathrm{MeV} / \mathrm{nucl})^{-1}$

where $C=9.42 \times 10^{4}$ is a normalization constant, and $E_{0}$ is a form parameter which is between 0 and $940 \mathrm{MeV}$. Changes in $E_{0}$ will change the spectra of low-energy cosmic rays substantially but have almost no effect for the high-energy end. Smaller values of $E_{0}$ represent more low-energy cosmic-ray particles (see Fig. 1). Webber \& Yushak (1983) found that $E_{0}=300 \pm 100 \mathrm{MeV}$ can explain the measured ${ }^{3} \mathrm{He} /{ }^{4} \mathrm{He}$ ratio and their observed spectra very well. The energy spectra for different values of $E_{0}$ are shown in Fig. 1.

The COMPTEL observations of broad gamma-ray lines of a few $\mathrm{MeV}$ from the Orion complex (Bloemen et al. 1994) suggest an unusually high flux of $\sim 10-100 \mathrm{MeV} \mathrm{C}$ and $\mathrm{O}$ nuclei in this region and a rather flat energy spectrum (Ramaty 1996) which is estimated to be several times the normal Galactic cosmic-ray components of these nuclei (Kozlovsky et al. 1997). This observation of low-energy gamma rays from a possibly enhanced intensity of $10-100 \mathrm{MeV}$ nucleon ${ }^{-1} \mathrm{C}$ and $\mathrm{O}$ nuclei indicates that such regions associated with star-formation are possible sources for the low-energy cosmic rays.

The energy spectra given by Webber \& Yushak (1983) are somewhat lower than the energy spectra given by 
Table 1. Galactic cosmic-ray (GCR) source abundances compared to the Solar abundances (normalized to $H \equiv 1.0 \times 10^{6}$ ).

\begin{tabular}{lllll}
\hline \hline$Z$ & Elem. & GCRs $^{1}$ & Solar $^{2}$ & $E_{\max }(\mathrm{MeV} / \text { nucl })^{3}$ \\
\hline 1 & $\mathrm{H}$ & $1.0 \times 10^{6}$ & $1.0 \times 10^{6}$ & - \\
2 & $\mathrm{He}$ & $6.9 \times 10^{4}$ & $9.8 \times 10^{4}$ & 1.4 \\
6 & $\mathrm{C}$ & 3000. & 355. & 22.5 \\
8 & $\mathrm{O}$ & 3720. & 741. & 45.8 \\
12 & $\mathrm{Mg}$ & 734. & 38.0 & 132.6 \\
14 & $\mathrm{Si}$ & 707. & 36.3 & 209.8 \\
26 & $\mathrm{Fe}$ & 713. & 31.6 & $\infty$ \\
\hline
\end{tabular}

1 - Meyer et al. (1998); 2 - Grevesse et al. (1996).

$3-E_{\max }$ is the maximum energy which can heat a $0.1 \mu \mathrm{m}$ grain up to $27 \mathrm{~K}$ by a cosmic ray ion.

Ip \& Axford (1985). Because of the big uncertainties in the measurements and the calculations, this may not be significant; however, the spectra in the low energy range would also be much higher than the spectra given in these two papers as indicated by the COMPTEL data (Bloemen et al. 1994). Ip \& Axford (1985) only took supernova explosions as the cosmic ray source, but there are also other sources available such as the ambient interstellar medium and stellar flares (Eichler 1980; Cowsik 1980; Fransson \& Epstein 1980). Therefore, the cosmic ray flux at low energies is still full of uncertainties. We will use the approximation given by Webber \& Yushak (1983) because it is easy to adopt and in good agreement with the older observations. However, we will allow some variations in the low energy range, i.e., increase the flux by a factor of 2-5 (smaller $E_{0}$ in practice) to see the consequences on quantities such as the cosmic ray ionization rate and the UV radiation field in the dense clouds as well as the resulting physical and chemical structure.

The elemental composition of the cosmic ray nuclei depends on the composition of the source and on the propagation through the interstellar medium. The elemental abundances of the most important cosmic-ray particles are listed in Table 1. It is clear that these are quite different from the elemental abundances in the solar system. From the measurements (Simpson 1983), it is seen that the velocity distributions of heavy particles are very similar to those of protons, with helium the exception in the low energy range. It is assumed here that they are exactly the same, which should not be a bad estimate. The differential flux of protons, carbon, oxygen and iron ions under such assumptions is shown in Fig. 2.

\section{Dust models}

The UV photon flux in different regions of the cloud is the key factor affecting the radical production in the ice mantle and will be discussed in detail in the following sections. To determine the UV field in the cloud, the scattering parameters in the UV region are needed. Because the scattering properties in the farultraviolet are difficult to determine from the observations, we will derive them theoretically from a dust model. In particular, the grain albedo $\omega_{\lambda}$, the Henyey-Greenstein asymmetry factor $g_{\lambda}$ and the extinction curve $A(\lambda) / A(V)$ are of great importance in determining the radiation field. Here we use the dust model

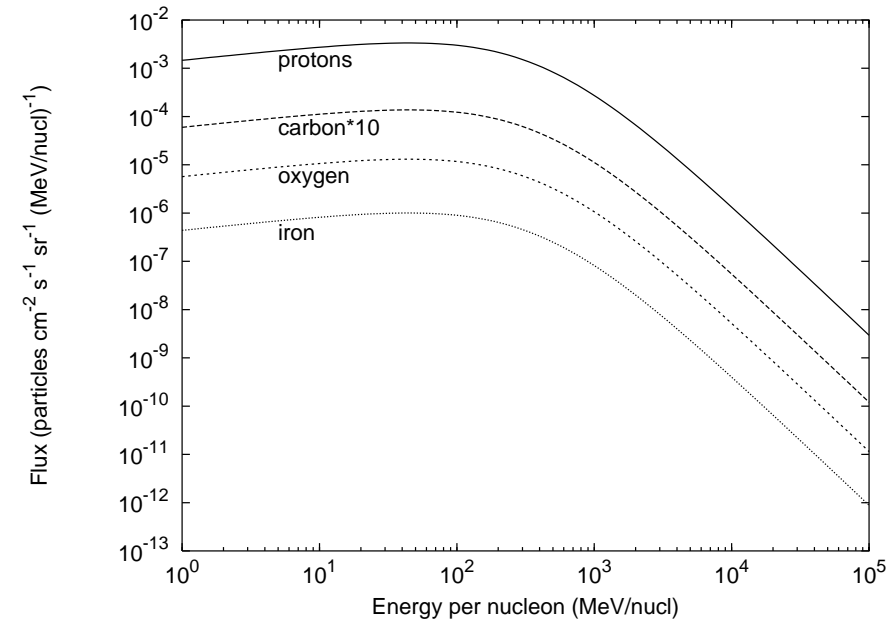

Fig. 2. Differential flux of protons, carbon, oxygen and iron in the energy range from $1 \mathrm{MeV}$ to $10^{5} \mathrm{MeV}$ using $E_{0}=400 \mathrm{MeV}$. The line for carbon is multiplied by a factor of 10 for clarity.

by Li \& Greenberg (1997), which is a trimodal dust model: large silicate core-organic refractory mantle dust particles; very small carbonaceous particles responsible for the hump extinction; and PAH's responsible for the far-ultraviolet extinction. The sizes, numbers, masses and volumes of the three dust components of this model are given in Table 2. Such a model satisfies the observations for both the extinction curve and polarization as well as the cosmic abundances. Because the polarization is not taken into account in our calculations, we can use the equivalent spherical particles instead of the finite cylinder used by Li \& Greenberg (1997), which makes the calculations much easier with enough accuracy.

Because the dust grains accrete ice and small particles in the denser region of the cloud at temperatures lower than $20 \mathrm{~K}$, the properties of the dust inside dense clouds are quite different from those at the cloud edge. In order to investigate such effects, we consider three different situations: 1) diffuse cloud dust without any accretion; 2) $50 \%$ of the adopted $\mathrm{H}_{2} \mathrm{O}$, hump particles and PAHs (see Table 2) are accreted onto the core-mantle dust particles; 3 ) all the $\mathrm{H}_{2} \mathrm{O}$, hump particles and PAHs are accreted onto the core-mantle dust particles. They are assumed to accrete onto the grain at the same percentage. Observations show that the relative abundance of water ice is about $1.4 \times 10^{-4}$ per hydrogen nucleus (Schutte 1998). We assume that this value corresponds to the maximum available amount of $\mathrm{H}_{2} \mathrm{O}$ molecules that can accrete on the core-mantle grains together with hump particles and PAHs. The adopted mass density of the silicate cores is $3.5 \mathrm{~g} \mathrm{~cm}^{-3}$, the organic refractory mantle $1.8 \mathrm{~g} \mathrm{~cm}^{-3}$, the hump particles $2.3 \mathrm{~g} \mathrm{~cm}^{-3}$ and the PAHs $2.4 \times 10^{-7} \mathrm{~g} \mathrm{~cm}^{-3}$ (Li \& Greenberg 1997). Therefore, a mean density for such a grain of $1.47 \mathrm{~g} \mathrm{~cm}^{-3}$ and a typical radius as $0.14 \mu \mathrm{m}$ are obtained if half of the water and small particles have accreted onto the grain surface in the form of ice.

Following the method employed by Li \& Greenberg (1997), the albedo $\omega$, asymmetry factor $g$ and the extinction curve $A(\lambda) / A(V)$ are calculated for the three different kinds of dust grains (Fig. 3). In order to compare the extinction curves, all 
Table 2. Sizes $(a)$, numbers $(n)$, masses $(m)$ and volumes $(V)$ of each dust component in the trimodal dust model (Li \& Greenberg 1997) together with the maximum $\mathrm{H}_{2} \mathrm{O}$ in the ice mantles.

\begin{tabular}{lllll}
\hline \hline & core-mantle $(\mathrm{cm})$ & hump & PAH & $\mathrm{H}_{2} \mathrm{O}$ \\
\hline size & $n(a) \sim \exp \left[-5\left(\frac{a-a_{c}}{a_{i}}\right)^{q}\right]$ & $n(a) \sim a^{-r}$ & $n(a) \sim a^{-r}$ & \\
distribution & $a_{c}=0.070 \mu \mathrm{m}, a_{i}=0.066 \mu \mathrm{m}, q=2$ & $a \in[15,120] \AA, r=3$ & $a \in[6,15] \AA, r=3$ & \\
\hline$n / n_{\mathrm{H}}$ & $3.89 \times 10^{-13}$ & $2.03 \times 10^{-9}$ & $3.11 \times 10^{-7}$ & $1.4 \times 10^{-4(1)}$ \\
$m / m_{\mathrm{cm}}$ & 1.00 & 0.11 & 0.12 & 1.01 \\
$V / V_{\mathrm{cm}}$ & 1.00 & 0.2336 & 0.2548 & 2.394 \\
\hline$(1)-$ Schutte (1998). & & &
\end{tabular}

curves are normalized to the visual extinction without accretion as unity. It is clear that as the grains accumulate mantles, their albedo increases and there is also a small increase in asymmetry factor, but the biggest enhancement is in the visual and infrared region for the extinction curve compared with the extinction curve of the dust grains at the edge of the cloud which have no such ice mantle. The big difference for the extinction curve between the grains with ice mantles and those without ice mantles is due to the reduction of small particles which become part of the mantle of the big grain.

Teixeira \& Emerson (1999) showed that there are threshold extinctions below which the $\mathrm{H}_{2} \mathrm{O}$ mantles cannot survive and this threshold is around $A_{\mathrm{V}}=3 \mathrm{mag}$ in the case of the Taurus cloud. There is also a linear correlation between the column density of water ice and the visual extinction for $A_{\mathrm{V}}>3$ mag. This points to fast accretion of water ice onto the dust grains in the clouds around $A_{\mathrm{V}}=3 \mathrm{mag}$. We assume no ice mantle when $A_{\mathrm{V}} \leq 3$ mag and a constant $\mathrm{H}_{2} \mathrm{O}$ ice abundance whenever $A_{\mathrm{V}}>3 \mathrm{mag}$.

\section{Ultraviolet photons inside dense clouds}

\subsection{Cosmic-ray ionization rate}

When cosmic-ray particles travel in the interstellar medium, they lose their energy by exciting and ionizing atoms. For dense clouds, the interstellar UV field cannot penetrate into the dense regions of the clouds $\left(A_{\mathrm{V}} \gtrsim 5 \mathrm{mag}\right)$ due to the absorption and scattering by dust. Thus the cosmic ray ionization and the cosmic-ray-induced UV field (Prasad \& Tarafdar 1983) are thought to be the sole drivers for the gas phase and grain surface chemistry in this kind of region. Both of these processes are characterized by the cosmic-ray ionization rate. In order to get the primary cosmic ray ionization rate $\zeta_{\mathrm{p}}$, the distribution of the primary electron energy spectrum produced by the interaction of cosmic-ray particles (mostly protons) and the interstellar medium (mainly molecular hydrogen and helium) needs to be known:

$\zeta_{\mathrm{p}}=\int \frac{\mathrm{d} n_{\mathrm{e}}}{\mathrm{d} W} \mathrm{~d} W$

and such a spectrum is given by

$\frac{\mathrm{d} n_{\mathrm{e}}}{\mathrm{d} W}=\int_{M W / 4 m}^{\infty} \frac{\mathrm{d} \sigma}{\mathrm{d} W} \frac{\mathrm{d} n}{\mathrm{~d} E} \mathrm{~d} E$.

Here, $\mathrm{d} \sigma / \mathrm{d} W$ is the differential cross section for producing an electron of energy $W$ by proton impact on molecular hydrogen,

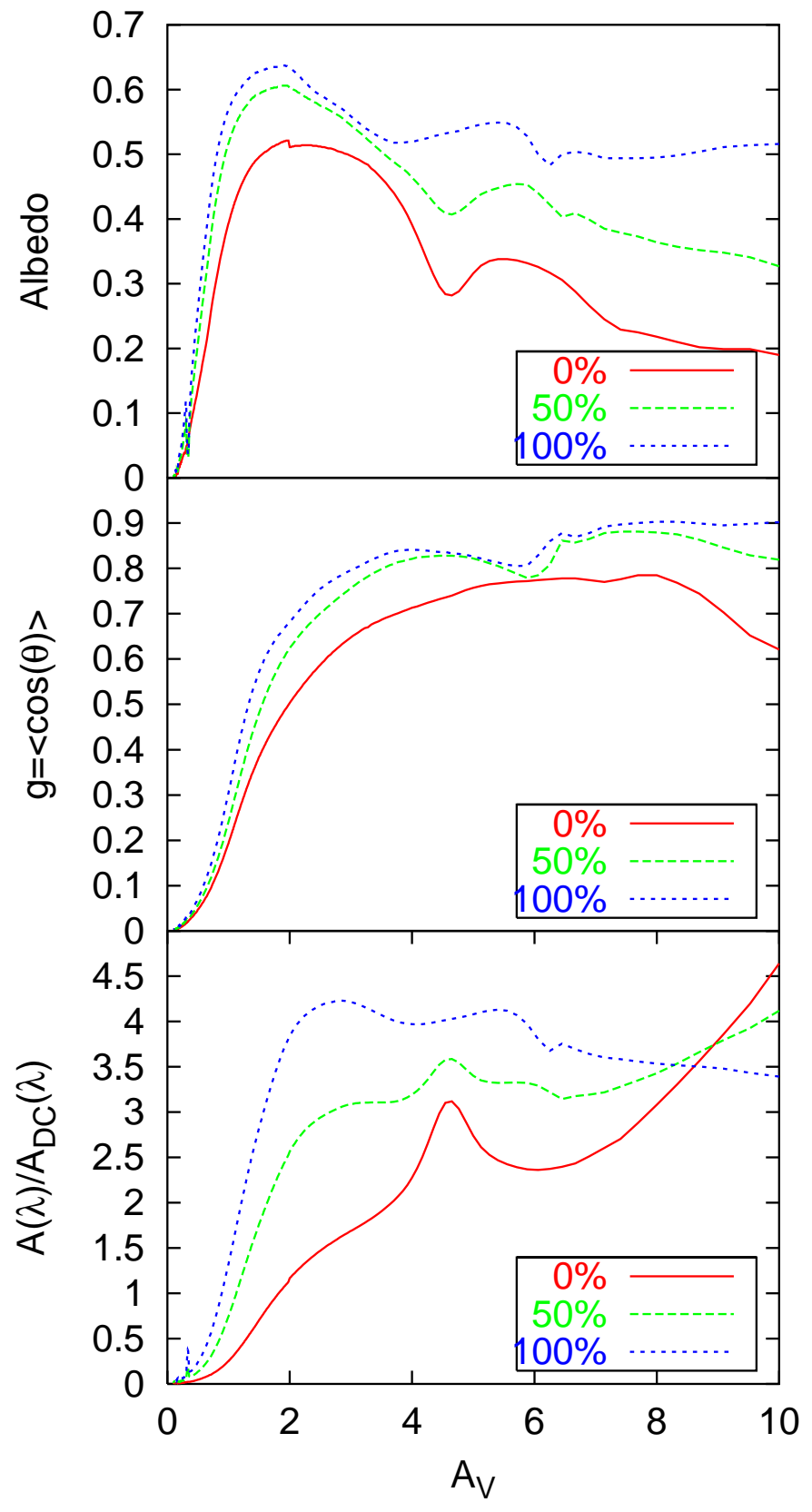

Fig. 3. The albedo, asymmetry factor and extinction curve for three kinds of dust: the grains at the edge of the cloud with no water ice, no hump particles and no FUV particles accreted onto the large coremantle grain, $50 \%$ of the small particles and water molecules accreted on the large grains and $100 \%$ of the small particles and water molecules are accreted 
$M / m$ is the mass ratio between proton and electron and $\mathrm{d} n / \mathrm{d} E$ is the cosmic ray proton flux. The differential cross-section for ionization is given by the relation (Opal et al. 1971)

$\frac{\mathrm{d} \sigma(E, W)}{\mathrm{d} W}=\frac{F(E)}{1+\left(W / W_{0}\right)^{2.1}}$,

where $E$ is the energy of the incident particles, $W$ is the energy of the ejected electron, and $F(E)$ is a normalization factor defined as

$F(E)=\frac{\sigma(E)}{W_{0} \arctan \left[\left(E-E_{\mathrm{I}}\right) / 2 W_{0}\right]}$,

where $W_{0}$ is a shape parameter which is $8.3 \mathrm{eV}$ for molecular hydrogen, and $E_{\mathrm{I}}$ is the ionization potential which is $15.4 \mathrm{eV}$ for molecular hydrogen. In the above equation, $\sigma(E)$ is the cross section for ionizing collisions due to proton impact with molecular hydrogen, which is given by Rudd et al. (1985) as

$\sigma=\left(\sigma_{l}^{-1}+\sigma_{h}^{-1}\right)^{-1}$

$\sigma_{h}=4 \pi a_{0}^{2}\left[p_{1} \ln (1+x)+p_{2}\right] / x$

$\sigma_{l}=4 \pi a_{0}^{2} p_{3} x^{p_{4}}$

where $a_{0}=0.529 \AA, x=m_{\mathrm{e}} v^{2} / E_{\mathrm{I}}=E_{\mathrm{p}} / 1836 E_{\mathrm{I}}$. For molecular hydrogen $E_{\mathrm{I}}=15.4 \mathrm{eV}, p_{1}=0.71, p_{2}=1.63, p_{3}=0.51$ and $p_{4}=1.24$.

It remains to estimate the contribution of the heavy nuclei cosmic rays to ionize molecular hydrogen. As discussed in Sect. 2, the velocity distribution of heavy particles follow that of protons. Therefore, we can just multiply with a correction factor to take their effect into account:

$\eta=\sum_{k} A_{k} Z_{k}^{2}$

where $A_{k}$ is the relative number of nuclei with charge $Z_{k} e$ in the cosmic-ray particles. Although such assumptions are not completely valid, they can give a rough estimate of the contribution of heavy particles. Using the values of $A_{k}$ listed in Simpson (1983), we obtain $\eta \simeq 1.8$.

In a weakly ionized gas, which is the case in the dense cloud interior, secondary electrons also lose energy in ionization and excitations. The number of secondary ionizations in $\mathrm{H}_{2}$ is about 0.7 for each primary ionization (Cravens \& Dalgarno 1978). It is also necessary to take the small modification arising from the ionization of helium in the interstellar gas into account with an assumed ratio of about 0.1 (Cecchi-Pestellini \& Aiello 1992). Because of the high threshold, the second ionization of helium is rare. Therefore, an estimate of the total ionization rate should be

$\zeta=\eta(1+0.7+0.1) \zeta_{\mathrm{p}}$

\subsection{Cosmic ray induced photons}

Collisions of $\mathrm{H}_{2}$ with cosmic-ray particles and secondary electrons can produce excited $\mathrm{H}_{2}$ in the $B^{1} \Sigma_{u}^{+}$and $C^{1} \Pi_{u}$ states and these states instantaneously undergo radiative decay in lines of the Lyman and Werner system which produce UV photons in molecular clouds (Prasad \& Tarafdar 1983). This mechanism can be very important both in the physics and chemistry of the clouds where the diffuse galactic radiation field cannot penetrate efficiently. The resulting spectrum is quite complicated consisting of many individual lines. It is, however, sufficient for our purpose to neglect the individual lines by smoothing the spectrum over a sufficiently large wavelength interval (Roberge 1990).

The source function in a particular Lyman or Werner line can be expressed as:

$S^{*}(\lambda)=\frac{1}{4 \pi} \frac{n\left(\mathrm{H}_{2}\right)}{\alpha_{\mathrm{d}}(\lambda)} \zeta \varphi(\lambda)$

and

$\alpha_{\mathrm{d}}(\lambda)=\alpha_{\mathrm{d}}(V) \frac{A(\lambda)}{A(V)}$

where $n\left(\mathrm{H}_{2}\right)$ is the density of $\mathrm{H}_{2}$ molecules, $\alpha_{\mathrm{d}}$ the dust extinction per $\mathrm{H}_{2}$ molecule, and $\varphi(\lambda)$ the line emission profile. The total number of photons emitted in all the Lyman and Werner transitions is about 0.3 per primary cosmic ray ionization (Prasad \& Tarafdar 1983; Cravens et al. 1975). As mentioned above, we smooth it between $850 \AA$ to $1750 \AA$. We define the smoothed value as $\bar{\varphi}=0.3 / 900$. Then Eq. (11) can be written as:

$S^{*}(\lambda)=\frac{1}{4 \pi} \frac{N\left(\mathrm{H}_{2}\right) / A(V)}{E(\lambda)} \zeta \bar{\varphi}$

where $N\left(\mathrm{H}_{2}\right) / A_{\mathrm{V}}$ is taken equal to $1.0 \times$ $10^{21} \mathrm{~cm}^{-2} \mathrm{mag}^{-1}$ (Bohlin et al. 1978).

\subsection{Radiative transfer in dense clouds}

An evaluation of the radiation field inside a dusty cloud requires solution of the radiative transfer equation involving dust scattering. The problem of radiative transfer inside interstellar clouds has been studied by a number of authors. The spherical harmonic method has proved to be a good way to treat such transfer problems with dust scattering in homogeneous media (Flannery et al. 1980; Roberge 1983). We solve the radiative transfer equations in a plane-parallel cloud with embedded photon sources due to cosmic-ray $\mathrm{H}_{2}$ interaction (Prasad \& Tarafdar 1983) by expressing the specific intensity as a truncated series in Legendre polynomials.

In a plane-parallel cloud the specific intensity of radiation $I(\tau, \mu)$ is the solution of the radiative transfer equation,

$\mu \frac{\partial I(\tau, \mu)}{\partial \tau}=I(\tau, \mu)-S(\tau, \mu)$

where $\tau$ is the extinction optical depth and $\mu$ is the angel cosine with respect to the direction of decreasing $\tau$. The opacity is assumed to be due to the dust scattering in this paper, and is taken to be coherent, nonconservative and anisotropic so that the specific intensity does not depend on wavelength. By expanding $I(\tau, \mu)$, the embedded source $S^{*}(\tau, \mu)$ and the phase function with Legendre polynomials and truncating the series at a finite odd order of $L\left(P_{\mathrm{L}}\right.$ approximation), the equation of transfer is reduced to a set of differential equations (Roberge 1983)

$$
\begin{gathered}
l f_{l-1}^{\prime}+(l+1) f_{l+1}^{\prime}-(2 l+1)\left(1-\omega \sigma_{l}\right) f_{l}+(2 l+1) s_{l}=0 \\
(l=0,1, \ldots, L)
\end{gathered}
$$




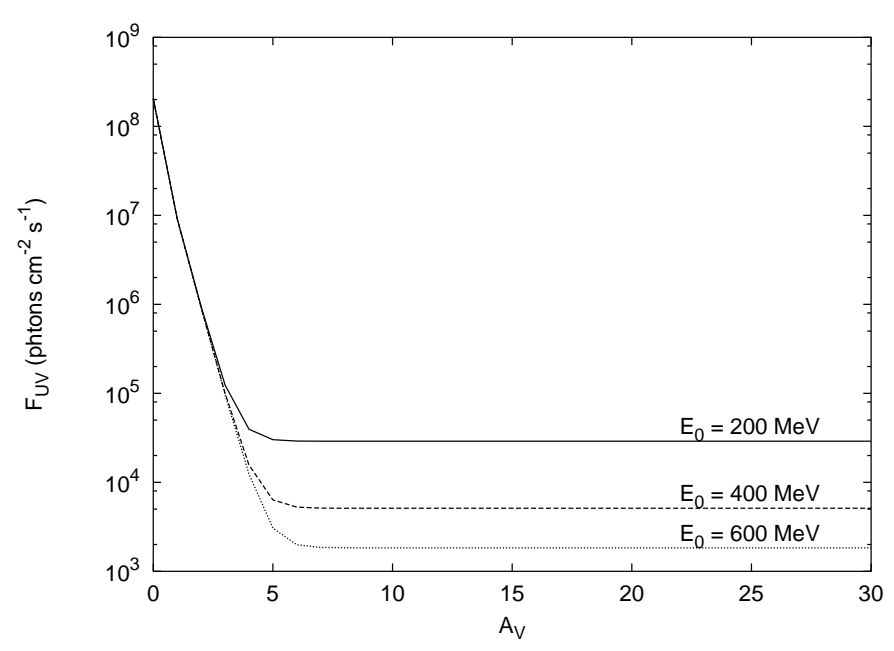

Fig. 4. The UV field in a dense cloud with the center visual extinction $A_{\mathrm{V}}=30$ mag for the different cosmic-ray spectra. $E_{0}$ is the form parameter as in Eq. (1). The incident field at the cloud edge is the standard ISRF as given by Mathis et al. (1983).

Roberge (1983) solved this set of equations with embedded sources with isotropic emission. The results are as follows:

$$
\begin{aligned}
I(\tau, \mu) & =\sum_{l=0}^{L}(2 l+1) f_{l}(\tau) P_{l}(\mu) \\
f_{l}(\tau) & =f_{l}^{+}(\tau)+(-1)^{l} f_{l}^{-}(\tau), \\
f_{l}^{+}(\tau) & =\sum_{m=1}^{M} R_{l m}\left\{C_{m} \exp \left[k_{m}\left(\tau-2 \tau_{\mathrm{c}}\right)\right]+\hat{W}_{m}(\tau)\right\} \\
f_{l}^{-}(\tau) & =\sum_{m=1}^{M} R_{l m}\left[C_{-m} \exp \left(k_{m} \tau\right)+\hat{Z}_{m}(\tau)\right],
\end{aligned}
$$

where $P_{l}(\mu)$ are the Legendre polynomials, $\tau_{\mathrm{c}}$ is the central optical depth and $k_{m}$ and $\boldsymbol{R}$ are the eigenvalues and the matrix of corresponding eigenvectors for Eq. (15) (see Roberge 1983, for details). The functions $\hat{W}$ and $\hat{Z}$ are as follows for isotropic embedded sources:

$$
\begin{aligned}
& \hat{W}_{m}(\tau)=\frac{S^{*}}{1-\omega}\left(R^{-1}\right)_{m, 0}\left\{1-\exp \left[-k_{m}\left(2 \tau_{\mathrm{c}}-\tau\right)\right]\right\} \\
& \hat{Z}_{m}(\tau)=\frac{S^{*}}{1-\omega}\left(R^{-1}\right)_{m, 0}\left[1-\exp \left(-k_{m} \tau\right)\right]
\end{aligned}
$$

where $S^{*}$ is the embedded source function. For the interstellar radiation field, we use the analytical representation of the radiation mean intensity obtained by Mathis et al. (1983), which forms the boundary condition at $\tau=0$.

The UV flux in dense clouds can be obtained from the specific intensity of radiation $I$ as:

$$
F_{\mathrm{UV}}(\tau)=\int I(\tau, \mu) \mu \mathrm{d} \Omega
$$

Figure 4 shows a calculation of the UV field in a dense cloud with a central visual extinction of 30 mag using the methods discussed above.

\section{Energy deposition to the grain}

\subsection{Grain heating by cosmic ray particles}

When a cosmic ray particle collides with a dust grain, it will deposit energy into the grain, and such energy will partly go to heat the grain. Léger et al. (1985) made an approximation for the volumic specific heat of interstellar grains:

$$
\begin{aligned}
C_{\mathrm{V}}(T) & =1.4 \times 10^{-4} T^{2} \mathrm{~J} \mathrm{~cm}^{-3} \mathrm{~K}^{-1} 10<T<50 \mathrm{~K} \\
& =2.2 \times 10^{-3} T^{1.3} \mathrm{~J} \mathrm{~cm}^{-3} \mathrm{~K}^{-1} 50<T<150 \mathrm{~K}
\end{aligned}
$$

and we will use this formula in our calculations. Therefore, the energy needed to heat a dust grain of radius $a$ to temperature $T$ is given as follows

$\Delta E(\mathrm{eV})=3.17 \times 10^{4}\left(\frac{a}{0.1 \mu \mathrm{m}}\right)^{3}\left(\frac{T}{30 \mathrm{~K}}\right)^{3} \quad T<50 \mathrm{~K}$.

From the above equation, it is found that $2.27 \times 10^{4} \mathrm{eV}$ is needed to heat a $0.1 \mu \mathrm{m}$ grain to $27 \mathrm{~K}$. The energy loss of a cosmic ray particle when passing a distance $\mathrm{d} s$ through material is given by the Bethe-Bloch formula (Fano 1963):

$$
\begin{aligned}
-\frac{\mathrm{d} Q(E)}{\mathrm{d} s}= & Z^{2} \frac{Z_{0}}{A} K(\beta)\left\{f(\beta)-\ln (I)_{\mathrm{eV}}-\frac{C}{Z_{0}}-\frac{1}{2} \delta\right\} \\
& \mathrm{MeV} \mathrm{g}^{-1} \mathrm{~cm}^{2}
\end{aligned}
$$

where

$$
\begin{aligned}
K(\beta) & =0.307 / \beta^{2} \\
f(\beta) & =\ln \left[1.022 \times 10^{6} /\left(1-\beta^{2}\right)\right]-\beta^{2} .
\end{aligned}
$$

In the above equations, $\beta$ is the velocity relative to the speed of light, $Z$ is the charge of the incident particles, $Z_{0} / A$ is the number of electrons per atomic weight of the material, $I$ is the mean excitation potential per electron of the material, $C / Z_{0}$ is the shell correction parameter and $\delta$ is the density effect correction. Figure 5 shows the result for such a calculation for an energetic iron particle passing a $0.1 \mu \mathrm{m}$ dust grain. It is seen that in the energy range of interest, $1-1000 \mathrm{MeV}$, the energy loss, which partly goes into heating the dust grain, decreases as the energy of the particle increases.

In order to determine how frequent a dust grain will undergo explosive release of its stored chemical energy, the time interval for cosmic ray particles heating a grain to $\geq 27 \mathrm{~K}$ (d'Hendecourt et al. 1982), which is the trigger temperature for free radical chain reactions, needs to be calculated. Because the energy deposition is proportional to the square of the incident particle charge $Z$ (see Eq. (24)), heavy ions in the cosmic rays play a crucial role in heating the grain. Léger et al. (1985) obtained such a time scale by using only Fe ions. In our calculation, several other heavy ions which can contribute to such heating are taken into account. These include $\mathrm{C}, \mathrm{N}$, $\mathrm{O}, \mathrm{Mg}, \mathrm{Si}$, and $\mathrm{Fe}$ which are the most abundant heavy ions in the cosmic rays. The abundant protons and alpha particles cannot deposit enough energy to heat a $0.1 \mu \mathrm{m}$ grain to $27 \mathrm{~K}$ (see Table 1). The rate of such events for a grain of radius $a$ is:

$t_{\text {heat }}^{-1}=\sum_{Z} R_{Z}=\sum_{Z} \pi a^{2} \int_{E_{\min }}^{E_{\max }} 4 \pi \frac{\mathrm{d} n}{\mathrm{~d} E} f_{Z} \mathrm{~d} E$, 


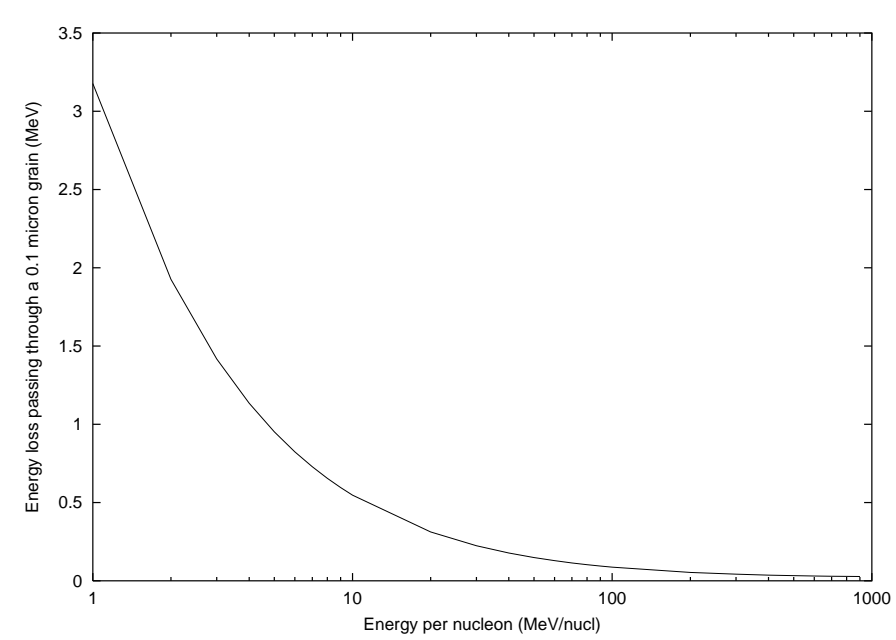

Fig. 5. The energy loss of an energetic cosmic ray iron particle when passing through a $0.1 \mu \mathrm{m}$ dust grain.

where $f_{Z}$ is the fraction of energy lost by heating the grain, $\frac{\mathrm{d} n}{\mathrm{~d} E}$ is the cosmic ray flux, $E_{\min }$ is the lowest energy needed to penetrate into the dense clouds and $E_{\max }$ is the maximum energy up to which the particle can heat a grain to $27 \mathrm{~K}$. $E_{\max }$ is a function of grain radius $a$. Table 1 gives values of $E_{\max }$ for the elements of interest. Here $\infty$ means that iron ions can deposit enough energy in the entire energy range. The rate of heating events is about twice that used by Léger et al. (1985). The reason for this difference, as outlined above, is that they exclusively relied on $\mathrm{Fe}$ nuclei to heat the grains, while we take all heavy ions into account in our calculation.

\subsection{Energy deposition to ice mantles by cosmic ray particles and by UV photons}

The source of the energy which is deposited in the ice mantles is crucial for understanding the processes occurring in ice mantles. The energy deposited by cosmic ray particles and that by absorbing UV photons are thought to be the two main contributors in dense shielded regions of clouds $\left(A_{\mathrm{V}} \gtrsim 5\right)$. The energy deposition to the water ice by all cosmic-ray particles is

$E_{\text {dep }}(\mathrm{CR})=\frac{1}{N_{\text {ice }}} \sum_{Z} 4 \pi \rho_{\text {ice }} \eta \int_{E_{-}}^{E_{+}} \frac{\mathrm{d} Q(E)}{\mathrm{d} s} \frac{\mathrm{d} n}{\mathrm{~d} E} f_{Z} \mathrm{~d} E$

in $\mathrm{eV}$ molecule $\mathrm{e}^{-1} \mathrm{~s}^{-1}$, where $\mathrm{d} Q(E) / \mathrm{d} s$ is the energy lost by a heavy ion passing through the water ice per centimeter, and $\rho_{\text {ice }}=1.0 \mathrm{~g} \mathrm{~cm}^{-3}$ is the density of the ice mantle. $\left[E_{-}: E_{+}\right]$is the energy range of the cosmic-ray spectrum. Here we use $E_{-}=$ $1 \mathrm{MeV}$ and $E_{+}=10^{4} \mathrm{MeV} . N_{\text {ice }} \approx 1 /\left(18 \times 1.66 \times 10^{-24}\right) \mathrm{cm}^{-3}$ is the number density of water ice.

To calculate the energy input via UV photons absorption, we assume the ice mantles are pure water ice and use the cross section $\sigma_{\mathrm{UV}}\left(\mathrm{H}_{2} \mathrm{O}\right) \approx 2.0 \times 10^{-18} \mathrm{~cm}^{2}$ (Okabe 1978). The UV field $F_{\mathrm{UV}}$ is obtained using the method discussed in the previous section.

$E_{\text {dep }}(\mathrm{UV})=\sigma_{\mathrm{UV}}\left(\mathrm{H}_{2} \mathrm{O}\right) F_{\mathrm{UV}} \hat{E}_{\text {photon }} \mathrm{eV}$ molecule ${ }^{-1} \mathrm{~s}^{-1}$

where $\hat{E}_{\text {photon }} \approx 6 \mathrm{eV}$ is the average energy of UV photons. The calculated results for cosmic-ray energy spectra with
Table 3. The energy deposition to water ice by cosmic rays and by UV photons for several cosmic-ray spectra with $E_{0}=200,400$ and $600 \mathrm{MeV}$.

\begin{tabular}{lll}
\hline \hline$E_{0}$ & $E_{\text {dep }}(\mathrm{CR})$ & $E_{\text {dep }}(\mathrm{UV})$ \\
$\mathrm{MeV}$ & $\mathrm{eV} \mathrm{molecule}^{-1} \mathrm{~s}^{-1}$ & $\mathrm{eV} \mathrm{molecule}{ }^{-1} \mathrm{~s}^{-1}$ \\
\hline 200 & $3.17 \times 10^{-14}$ & $3.47 \times 10^{-13}$ \\
400 & $6.16 \times 10^{-15}$ & $6.13 \times 10^{-14}$ \\
600 & $2.41 \times 10^{-15}$ & $2.20 \times 10^{-15}$ \\
\hline
\end{tabular}

$E_{0}=200,400$ and $600 \mathrm{MeV}$ are listed in Table 3. It is seen that the energy input by UV photons is about an order of magnitude higher than the energy input by cosmic ray particles. Therefore, from the energy input point of view, the cosmic-ray induced UV field is more important for the chemistry in the ice mantle.

\section{Radical formation on the grain surface}

When illuminated by ultraviolet radiation, free radicals containing potential chemical energy are formed inside the ice mantles. The radicals are stored in the ice due to dissociations of molecular bonds and eventual addition of the "hot" dissociation products to $\mathrm{CO}$ in the ice mantle (Schutte \& Greenberg 1991). In order to estimate the concentration of free radicals as a function of time, the UV flux in dense clouds $F_{\mathrm{UV}}$ (photons $\mathrm{cm}^{-2} \mathrm{~s}^{-1}$ ) is obtained from the radiative transfer calculation discussed above. The concentration of free radicals $\varepsilon$ can be written as:

$\varepsilon=\varepsilon_{\max }\left[1-\exp \left\{-\alpha R\left(\mathrm{H}_{2} \mathrm{O}\right)\left[\mathrm{H}_{2} \mathrm{O}\right] / \varepsilon_{\max }\right\}\right]$

where

$R\left(\mathrm{H}_{2} \mathrm{O}\right)=\sigma_{\mathrm{UV}}\left(\mathrm{H}_{2} \mathrm{O}\right) F_{\mathrm{UV}} t$

Here $\varepsilon_{\max }$ is the maximum radical concentration, $\alpha$ is the efficiency of free radical production by UV photons, $t$ is the time interval for grain heating by a cosmic-ray particle to at least $27 \mathrm{~K}$, and $\left[\mathrm{H}_{2} \mathrm{O}\right]$ is the concentration of $\mathrm{H}_{2} \mathrm{O}$ in the grain. $\varepsilon_{\max }$ was found to be $2.6 \times 10^{-2}$ for a $\mathrm{CO}$ concentration of $16 \%$ (Schutte \& Greenberg 1991). The storage capacity of the ice for radicals $\varepsilon_{\max }$ probably depends considerably on the ice composition, in particular the $\mathrm{CO}$ concentration. $\varepsilon_{\max }$ is therefore expected to be lower for ice having a lower $\mathrm{CO}$ concentration. For this reason we also made calculations using $\varepsilon_{\max }=0.01$. Schutte \& Greenberg (1991) derived $\alpha \sim 0.5$ for $\mathrm{H}_{2} \mathrm{O}$. Differences may exist between the situation in the interstellar medium and that in the laboratory. Therefore we conservatively adopted some lower values for $\alpha$ in our calculations as well.

\section{The desorption rates}

As discussed above, we can calculate the cosmic ray ionization rate, ultraviolet photon flux and then the heating time scale of a dust grain by a cosmic-ray particle from an assumed cosmic ray energy spectrum. Equation (1) was used with several values of $E_{0}$ from $200 \mathrm{MeV}$ to $600 \mathrm{MeV}$ for the cosmic-ray spectra as 
shown in Fig. 1. The spectra of species other than protons are scaled with the relative abundances in Table 1 (Sect. 2) and examples of spectra are shown in Fig. 2 for $E_{0}=400 \mathrm{MeV}$. When the cosmic-ray particles travel into the clouds, they lose their energy by collisions with the gas and the dust grains. The main loss mechanism is through the ionization of molecular hydrogen. Léger et al. (1985) showed that such energy loss does not change the energy spectrum significantly as long as $A_{\mathrm{V}}<50$. Therefore, the cosmic-ray energy spectrum change due to the energy loss while passing through the cloud was neglected in the calculation.

Using the dust model discussed in Sect. 3, the radii of the grains are obtained in the different regions of the clouds, and then the time interval $t_{\text {heat }}$ to heat such a grain to a temperature of at least $27 \mathrm{~K}$ can be calculated using the cosmic ray spectrum. The radical concentration attained during this time interval can then be obtained using the UV flux calculated above by means of Eq. (27). There are two energy sources for heating the grain and then sublimating molecules from the grain surface. They are the cosmic-ray direct heating and the chemical energy released by the radical reactions. Radiative cooling and cooling by sublimation are the two main ways to remove excess energy from the grain. Schutte \& Greenberg (1991) showed that above $26 \mathrm{~K}$ the cooling by sublimation is dominant, while below $26 \mathrm{~K}$ radiative cooling plays the main role. Due to the exponential decay of the sublimation with temperature, it can be approximated that above $26 \mathrm{~K}$ the cooling is exclusively due to sublimation. For interstellar grains, the sublimation takes place primarily from the outer ice mantle consisting of the most volatile compounds such as $\mathrm{CO}$. Its sublimation rate at $26 \mathrm{~K}$ is about $10^{29}$ times higher than that of $\mathrm{CO}_{2}$, the next most volatile compound, and at $100 \mathrm{~K}$ around $10^{8}$ times. Therefore, the released chemical energy $E_{\text {chem }}$ and energy deposited by cosmic-ray collision $E_{\mathrm{CR}}$ minus the energy needed to heat the grain to $26 \mathrm{~K}$ $E_{26}$ are assumed to be consumed by $\mathrm{CO}$ sublimation. Therefore the rate coefficient for such desorption can be written as:

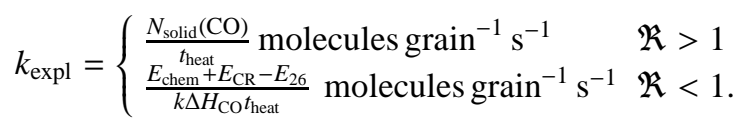

Here $\mathfrak{R}=\frac{\left(E_{\text {chem }}+E_{\mathrm{CR}}-E_{26}\right) / k \Delta H_{\mathrm{CO}}}{N_{\text {clid }}(\mathrm{CO})}$ is the ratio between the total solid $\mathrm{CO}$ that will be sublimated and the total solid $\mathrm{CO}$ on a grain. For $\Delta H_{\mathrm{CO}}$, we adopt the binding energy of $\mathrm{CO}$ on $\mathrm{CO}$ ice, $\Delta H_{\mathrm{CO}}=960 \mathrm{~K}$ (Sandford \& Allamandola 1990). This binding energy rather than that of $\mathrm{CO}$ on $\mathrm{H}_{2} \mathrm{O}$ ice is used because the desorption mechanisms are selective. At the beginning of the condensation, all atoms and molecules (only $\mathrm{H}_{2} \mathrm{O}$ and $\mathrm{CO}$ are shown in Fig. 6) likely condense onto the grain in mixed form, where also new species are formed through grain-surface reactions. When selective desorption such as that due to the release of chemical energy occurs, the most volatile molecules such as $\mathrm{CO}$ are desorbed and the molecules such as $\mathrm{H}_{2} \mathrm{O}$ still remain on the surface as the mantle. After the desorption, there is re-condensation. Because the desorption time scale is much shorter than the lifetime of the clouds, there are several rounds of desorption and condensation, which results in the onion structure of the ice mantles with the most volatile molecules in the outermost layer as shown in Fig. 6c.

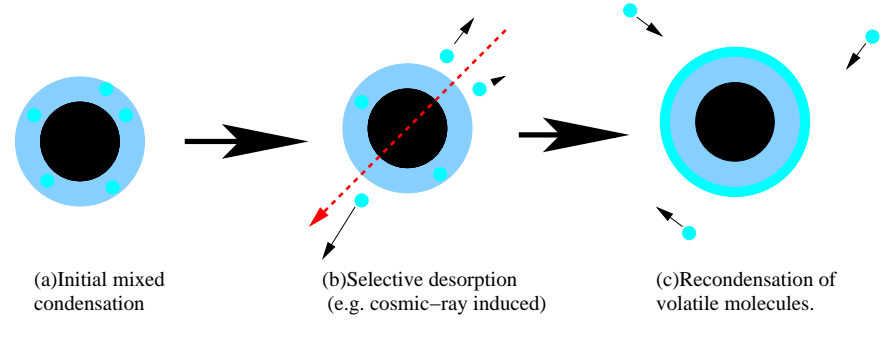

Fig. 6. Formation of layered ice mantles by selective desorption mechanisms.

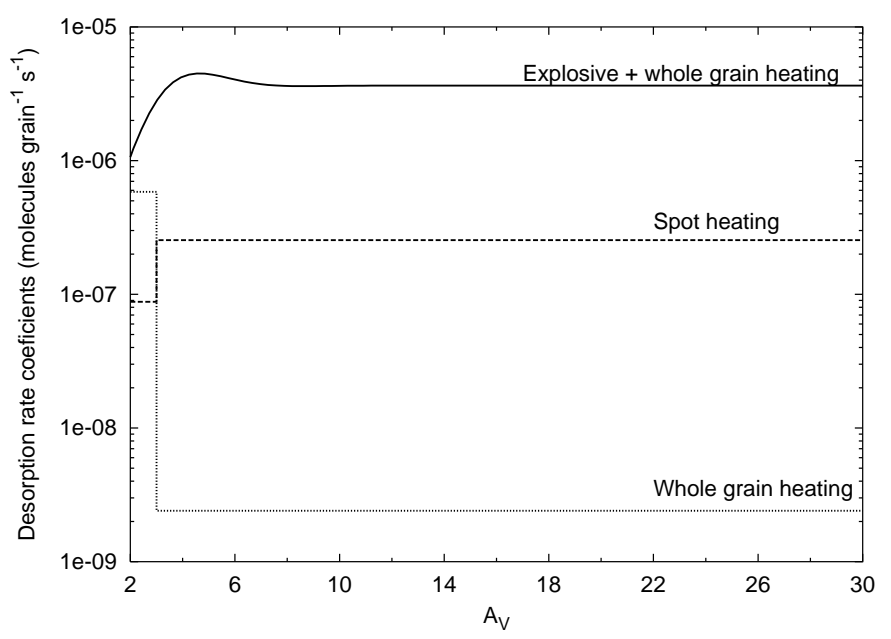

Fig. 7. Desorption rate coefficients as a function of visual extinction for spot heating, whole grain heating and chemical explosions.

Observations indeed confirm that in dense clouds most solid $\mathrm{CO}$ is embedded in an ice mantle which is dominated by $\mathrm{CO}$ itself (Chiar et al. 1995; Pontoppidan et al. 2003).

In Eq. (29), the whole grain heating is integrated in the rate coefficient calculation. Setting $E_{\text {chem }}=0$ will exclude the chemical energy release, thus the rate coefficients become equal to the rate by the whole grain heating.

Léger et al. (1985) proposed that the desorption due to cosmic-ray spot heating could play an important role for dust grains greater than $0.25 \mu \mathrm{m}$. The evaporation rate for $\mathrm{CO}$ by the spot heating was given by Léger et al. (1985) as $k_{\text {spot }}=$ 70 molecules $\mathrm{cm}^{-2} \mathrm{~s}^{-1}$ which is independent of the grain size. We take this desorption due to spot heating into account in the simulation. The calculated rates are shown in Fig. 7. The spotheating desorption rates increase due to the increased grain radius after accretion for $A_{\mathrm{V}} \geq 3$. However, the whole grain heating desorption rates decrease due to the larger radius. The rise of chemical explosive desorption rate (including whole grain heating desorption) is due to the increasing number of $\mathrm{CO}$ molecules in a grain ice mantle for $A_{\mathrm{V}} \lesssim 5$. The decrease of the rate for $A_{\mathrm{V}} \gtrsim 5$ is due to the decrease of the penetrating UV photons.

\section{Comparison with observations}

Using the desorption mechanism discussed above, we will model the observations of two dense clouds, L977 and IC 5146. 


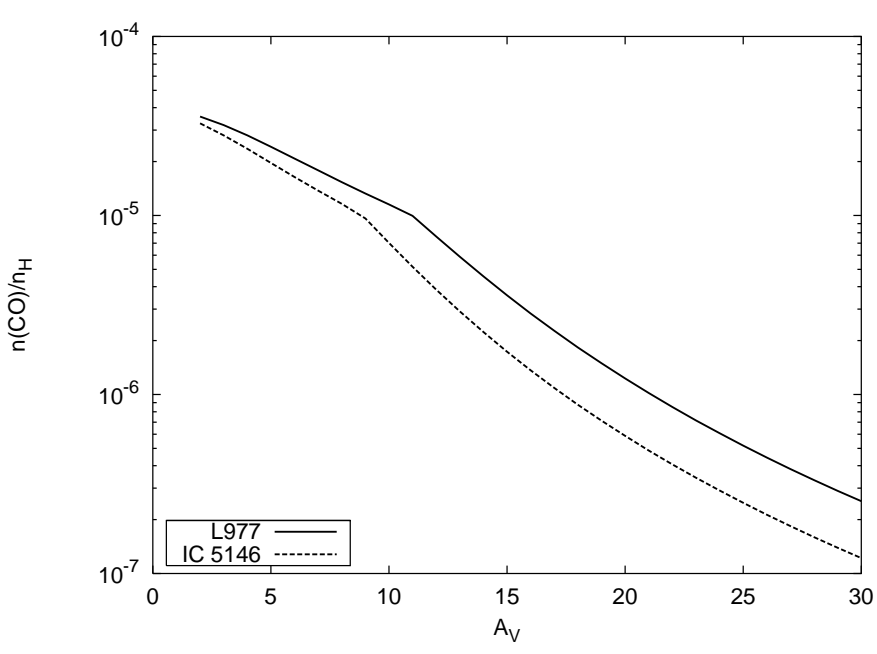

Fig. 8. The relative abundance of gaseous $\mathrm{CO}$ to total hydrogen as a function of visual extinction.

\section{1. $L 977$}

L977 is part of dark globular filament GF7 in the Cygnus complex and lies against a rich background of field stars toward the wrapped plane of the Galaxy. There is no obvious sign of ongoing star formation in this cloud. For the density structure of the cloud, we use the analysis in Alves et al. (1998), who show that the extinction (or column density) gradient in the cloud is nicely reproduced by a cylindrical geometry with $\rho(r) \propto r^{-2}$ in the range $2<A_{\mathrm{V}}<40$ mag (or roughly $1 \mathrm{pc}<r<0.1 \mathrm{pc}$ ). To be consistent with their results, which show a large systematic increase in volume density from low to high extinction, we adopt the following density profile: $n_{\mathrm{H}}=n_{0}\left(r / r_{0}\right)^{-2}=$ $10^{5}(r / 0.061 \mathrm{pc})^{-2}$. The density profile is in reasonable agreement with the average radial profile of extinction (Alves et al. 1998, Fig. 11). Alves et al. (1999) also observed the $J=(1-0)$ $\mathrm{C}^{18} \mathrm{O}$ emission line toward $\mathrm{L} 977$ to check the correlation between $\mathrm{C}^{18} \mathrm{O}$ emission and dust extinction. They found a linear correlation between the $\mathrm{C}^{18} \mathrm{O}$ column density and that of dust for cloud depths corresponding to $A_{\mathrm{V}} \lesssim 10 \mathrm{mag}$. For larger cloud depths, there is a notable break in the linear correlation. They suggested several causes to explain such a break, with the $\mathrm{CO}$ depletion the most likely one. Using the model discussed above, we seek to explain these observations.

Using the desorption rate obtained in Sect. 7 and taking the sticking coefficient for $\mathrm{CO}$ upon collision with a grain surface as unity, the gas phase $\mathrm{CO}$ abundance can be obtained as a function of visual extinction to the edge $A_{\mathrm{V}}$. In this simplest calculation, only the sticking onto and desorption of $\mathrm{CO}$ off the grain surface are considered to demonstrate the effect of the desorption. Because the total $\mathrm{CO}$ abundance is quite stable, the gas phase $\mathrm{CO}$ is only affected by the desorption and accretion. Thus, gas phase reactions and surface reactions can be excluded for simplicity. Figure 8 shows the relative abundance of gas-phase $\mathrm{CO}$ to total hydrogen $n_{\mathrm{H}}=n(\mathrm{H})+2 n\left(\mathrm{H}_{2}\right)$ varying with the visual extinction $A_{\mathrm{V}}$. At the edge of the cloud, most of the $\mathrm{CO}$ is in the gas phase due to the strong UV field coming from the outside as seen from Fig. 4. Going into the cloud, the relative abundance of gaseous $\mathrm{CO}$ decreases quickly due to

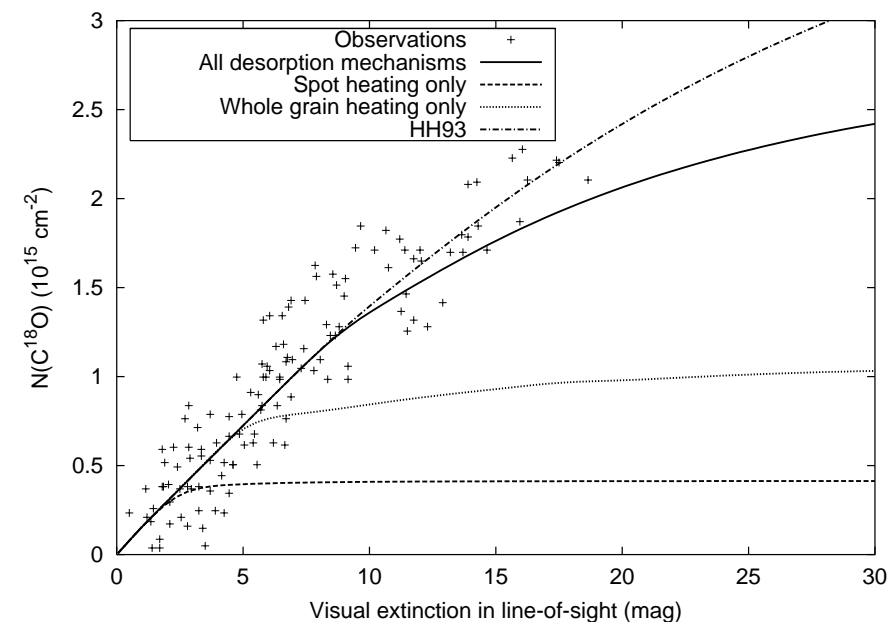

Fig. 9. The column density of $\mathrm{C}^{18} \mathrm{O}$ as a function of visual extinction $A_{\mathrm{V}}$ using the desorption due to cosmic-ray whole grain heating only (dotted line), cosmic-ray spot heating only (dashed line) and chemical energy released from free radicals combined with the other two (solid line). The points are the observations of L977 made by Alves et al. (1999). The dash-dotted line is the calculation using the desorption due to cosmic-ray heating by the Hasegawa \& Herbst (1993) approximation (see text for details).

the decreasing UV field and the increasing number density of the cloud. Because the accretion rate is proportional to the density of the molecule, the accretion rate increases as the visual extinction $A_{\mathrm{V}}$ increases. However, the desorption coefficient is proportional to the heating frequency. The heating time scale of a grain depends only on the cosmic-ray particle density and size of the grain, which is barely changed between the edge and the core of the cloud. The energy that will sublimate $\mathrm{CO}$ in the ice mantle includes the energy released via radical reactions and the energy deposited by a cosmic-ray particle passing through a grain particle minus the energy needed to heat the dust grain to $26 \mathrm{~K}$. This energy is almost constant with cosmic-ray energy spectrum when $A_{\mathrm{V}}>5$. As more and more $\mathrm{CO}$ molecules stick onto the ice mantle as $A_{\mathrm{V}}$ increases, the energy available is not enough to sublimate all the $\mathrm{CO}$ in the ice mantle, which means $\mathfrak{R}<1$ in Eq. (29). Therefore, the relative abundance of gas phase CO decreases faster when $\mathfrak{R}<1$, that is, solid CO is only partially sublimated for one heating event. Thus, in Fig. 8 it is seen that around $A_{\mathrm{V}}=10$, the relative abundance of $\mathrm{CO}$ decreases even faster.

We integrate the column density of $\mathrm{CO}$ along the line of sight and scale to $\mathrm{C}^{18} \mathrm{O}$ using $\left[\mathrm{CO} / \mathrm{C}^{18} \mathrm{O}\right]=560$ (Wilson \& Rood 1994) to compare our calculations with the observations. The calculated results are shown as the solid line in Fig. 9. They fit quite well with the observations for the dense region L977 (Alves et al. 1999). The adopted parameters are listed in Table 4. The model shows an apparent flattening at $A_{\mathrm{V}} \geq$ 25 mag caused by the depletion of CO (Fig. 8).

\subsection{IC 5146}

Similar studies have been performed for the dense core of the dark cloud IC 5146. Lada et al. (1999) investigated the 


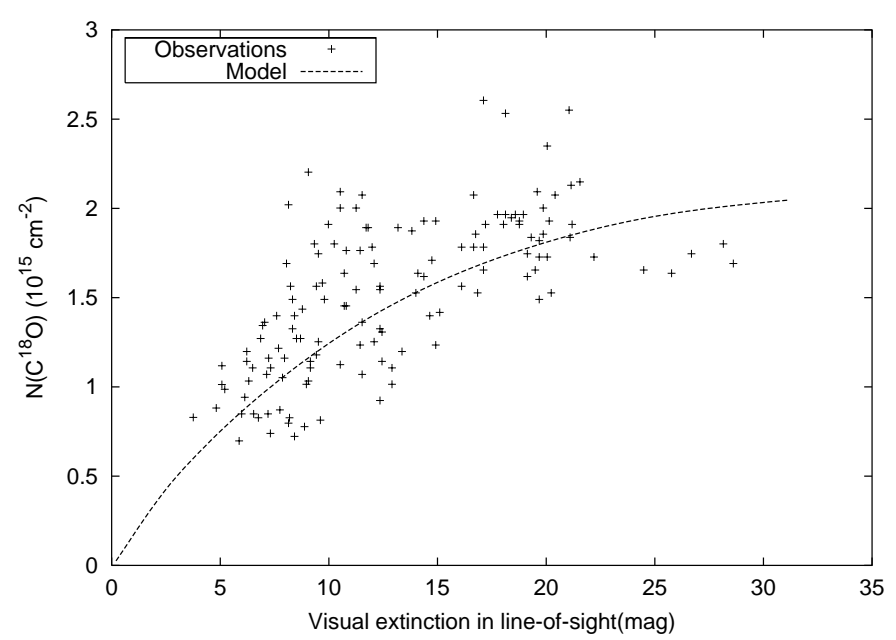

Fig. 10. The total $\mathrm{C}^{18} \mathrm{O}$ column density along the line of sight as a function of visual extinction for the dense core of IC 5146. The points are the observations of IC 5146 made by Kramer et al. (1999).

Table 4. Standard parameters used in the calculations

\begin{tabular}{ll}
\hline \hline Parameter & Value \\
\hline$E_{0}$ & $400 \mathrm{MeV}$ \\
$\alpha\left(\mathrm{H}_{2} \mathrm{O}\right)$ & 0.5 \\
$\Delta H(\mathrm{CO})$ & $960 \mathrm{~K}$ \\
$\sigma_{\mathrm{UV}}\left(\mathrm{H}_{2} \mathrm{O}\right)$ & $2.0 \times 10^{-18} \mathrm{~cm}^{2}$ \\
$E_{\mathrm{rad}}$ & $1.5 \mathrm{eV}$ \\
$n(\mathrm{CO}) / n_{\mathrm{H}}$ & $4 \times 10^{-5}$ \\
$\mathrm{CO} / \mathrm{C}^{18} \mathrm{O}$ & 560 \\
$k_{\text {spot }}$ & 70 molecules cm $\mathrm{cm}^{-1}$ \\
\hline
\end{tabular}

infrared extinction and the structure of the dense cloud associated with IC 5146 and find that the volume density profile is similar to L977, $n(r) \sim r^{-2}$ over a size scale $0.07<r<$ $0.40 \mathrm{pc}$ to reproduce the derived extinction gradient in the region. We adopt the following density profile: $n_{\mathrm{H}}=n_{0}\left(r / r_{0}\right)^{-2}=$ $10^{5}(r / 0.052 \mathrm{pc})^{-2}$, which is in good agreement with the radial profile of total column density (Fig. 8 in Lada et al. 1999). Using this density profile and the parameters in Table 4, the model can also fit the observations very well as seen in Fig. 10. The relatively smaller abundance of gas-phase CO in IC 5146 compared to that in L977 (Fig. 8) is due to the higher number density of the IC 5146 cloud. The model shows a flattening at $A_{\mathrm{V}} \geq 20 \mathrm{mag}$, which is consistent with the observational points.

\subsection{Parameters change}

We investigate the influence of varying the values of the model parameters on the calculated $\mathrm{CO}$ depletion. We compare these alternative results to the CO column density for IC 5146 to study which parameters are most constrained by the observations and to test the robustness of our model.

As discussed in Sect. 2, the energy spectra of cosmic-ray particles have considerable uncertainties. We investigate here the consequence of depletion due to different cosmic-ray spectra. Using Eq. (1), different cosmic-ray spectra can be obtained by varying $E_{0}$ (see Fig. 1 ). $E_{0}=200,400,600 \mathrm{MeV}$ are used for the model calculations keeping the other parameters the same as in Table 4. As seen in Fig. 1, smaller values of $E_{0}$ represent more low energy cosmic-ray particles and vice versa. It is clear that the desorption is much more efficient when more low energy cosmic-ray particles are available. There are two factors contributing to this result. On the one hand, because low energy cosmic-ray particles deposit more energy than high energy particles (see Fig. 5), more low energy cosmic-ray particles will heat the dust grain more frequently, which means that the heating time scale is shorter and thus the desorption more efficient. On the other hand, more low energy cosmic-ray particles can excite more molecular hydrogen which result in more UV photons in the region (see Fig. 4). Thus more free radicals are made at the same time on the grain surface. The desorption is enhanced as more energy is released to sublimate volatile molecules from the grain surface. The density profile of IC 5146 is adopted in the calculation. The results together with the observations for IC 5146 are shown in Fig. 11. It can be seen that the cosmic-ray energy spectrum has a prominent effect on the depletion of gas phase CO. We can try to constrain the cosmic-ray energy spectrum and thus the ionization rate from the observations. From Fig. 11, the best fit for the observations resulted in $E_{0} \approx 400 \mathrm{MeV}$, which means that the cosmic-ray ionization rate in this region is about $3.1 \times 10^{-17} \mathrm{~s}^{-1}$. This is consistent with the average value $(2.6 \pm 1.8) \times 10^{-17} \mathrm{~s}^{-1}$ derived by van der Tak \& van Dishoeck (2000).

As mentioned above in Sect. 6, the maximum radical concentration $\varepsilon_{\max }$ depends on the $\mathrm{CO}$ concentration in the ice mantle. The consequence of different $\varepsilon_{\max }$ are investigated in Fig. 12 for $\varepsilon_{\max }=0.026$ and $\varepsilon_{\max }=0.01$. It is seen that different $\varepsilon_{\max }$ only have a small effect on the column density of $\mathrm{CO}$ in the line of sight. This is because the radical concentration generally stays well below $\varepsilon_{\max }$ between subsequent cosmic ray heating events. Only when using very small values $\left(\varepsilon_{\max } \leq 0.001\right)$ will the desorption rate drop considerably.

The efficiency of free radical production by an ultraviolet photon $\alpha$ is also a source of uncertainties. Several values of $\alpha$ are used to investigate its effects. Figure 13 shows the results for similar calculations with $\alpha=0.5,0.25$ and 0.1 . It is seen that changing $\alpha$ has no effect on the column density for gas phase $\mathrm{CO}$ for $A_{\mathrm{V}}<15$, which is due to the fact that the available energy can sublimate all the $\mathrm{CO}$ in the ice mantle, i.e. $\mathfrak{R} \geq 1$ in Eq. (29). Some effects are found for the region $A_{\mathrm{V}}>15$, caused by the change of radical production.

The sticking coefficient $S_{\mathrm{CO}}$ of $\mathrm{CO}$ on a grain also affects the desorption of gaseous $\mathrm{CO}$. We investigate this effect with $S_{\mathrm{CO}}=1.0,0.5$ and 0.1 using the standard parameters as shown in Fig. 14. The effects of lowering the sticking coefficient are quite apparent, although they are smaller than those due to different cosmic-ray spectra (see Fig. 11). From Fig. 14b, it is seen that $S_{\mathrm{CO}}=1.0$ fits the obervations better than lower values since the curve becomes flat for $A_{\mathrm{V}}>23 \mathrm{mag}$. A sticking coefficient close to unity is consistent with recent theoretical studies by Al-Halabi et al. (2003). 

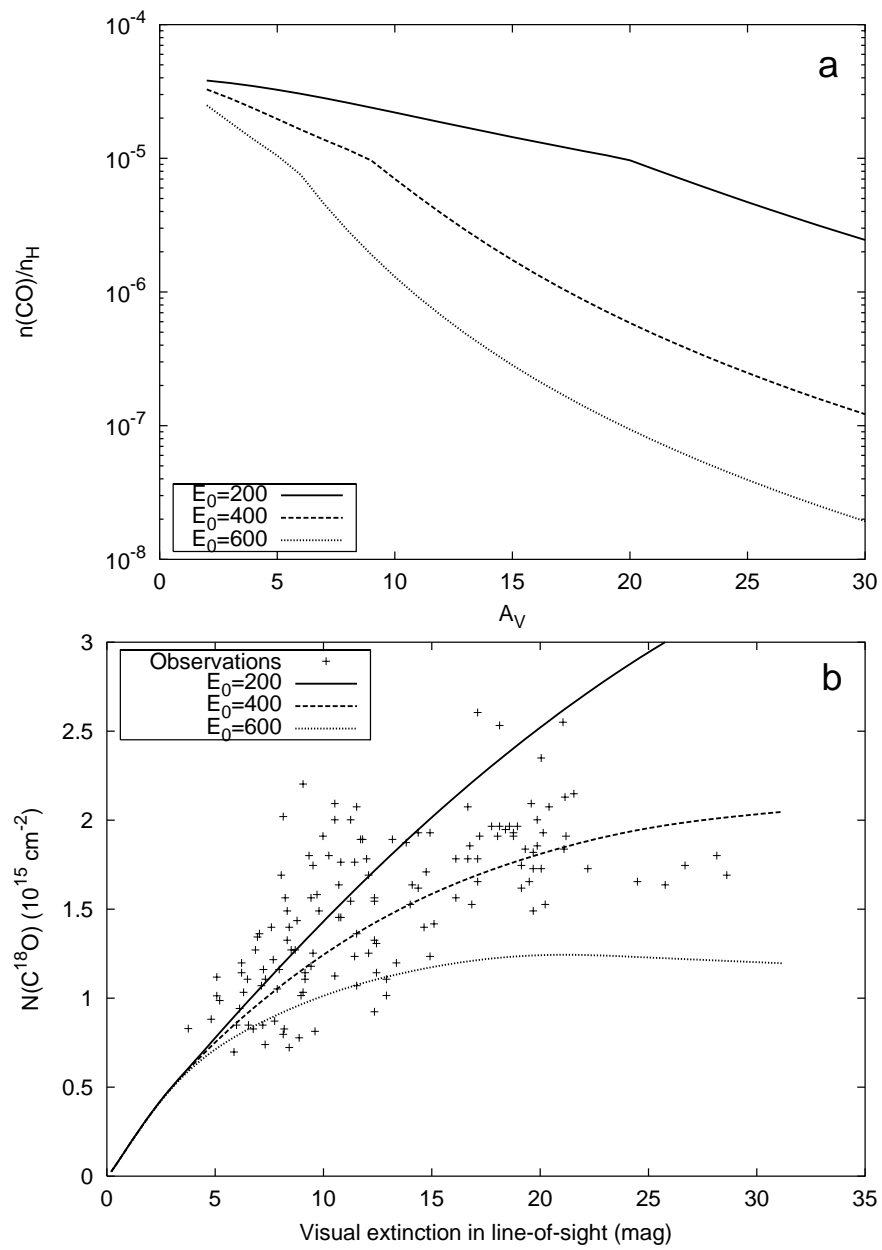

Fig. 11. The relative abundance of gaseous $\mathrm{CO}$ to total hydrogen a) and the $\mathrm{C}^{18} \mathrm{O}$ column density $\mathbf{b}$ ) as a function of visual extinction along the line-of-sight for different cosmic-ray energy spectra.

\section{Discussion}

There are three desorption routes related to the cosmic-ray particles traveling through a dust grain. They are the whole grain heating for the grains of medium size (around and less than $0.1 \mu \mathrm{m}$ ), spot heating for bigger grains and the release of chemical energy stored as radicals for grains with ice mantles. Separate calculations using only whole grain heating desorption, only spot heating desorption and all three mechanisms were performed with the standard parameters for L977 to compare the mechanisms (Sect. 8.1; Fig. 9). If the desorption due to cosmic-ray spot heating is the only way to desorb $\mathrm{CO}$ molecules from the ice mantles, it is impossible to maintain the observed gas phase CO abundance (see Fig. 9, dotted curve). This may imply that grains smaller than $0.25 \mu \mathrm{m}$ are dominant in dark clouds like L977, because the other two desorption mechanisms do not work for such big grains.

It is surprising to see that cosmic-ray induced whole grain heating is only effective at the edge of the cloud and is negligible inside, because Bergin et al. (1995) and Willacy \& Millar (1998) have shown that this desorption mechanism is effective throughout cloud cores. The cause of this discrepancy is the formulation of the desorption rate coefficient. In both
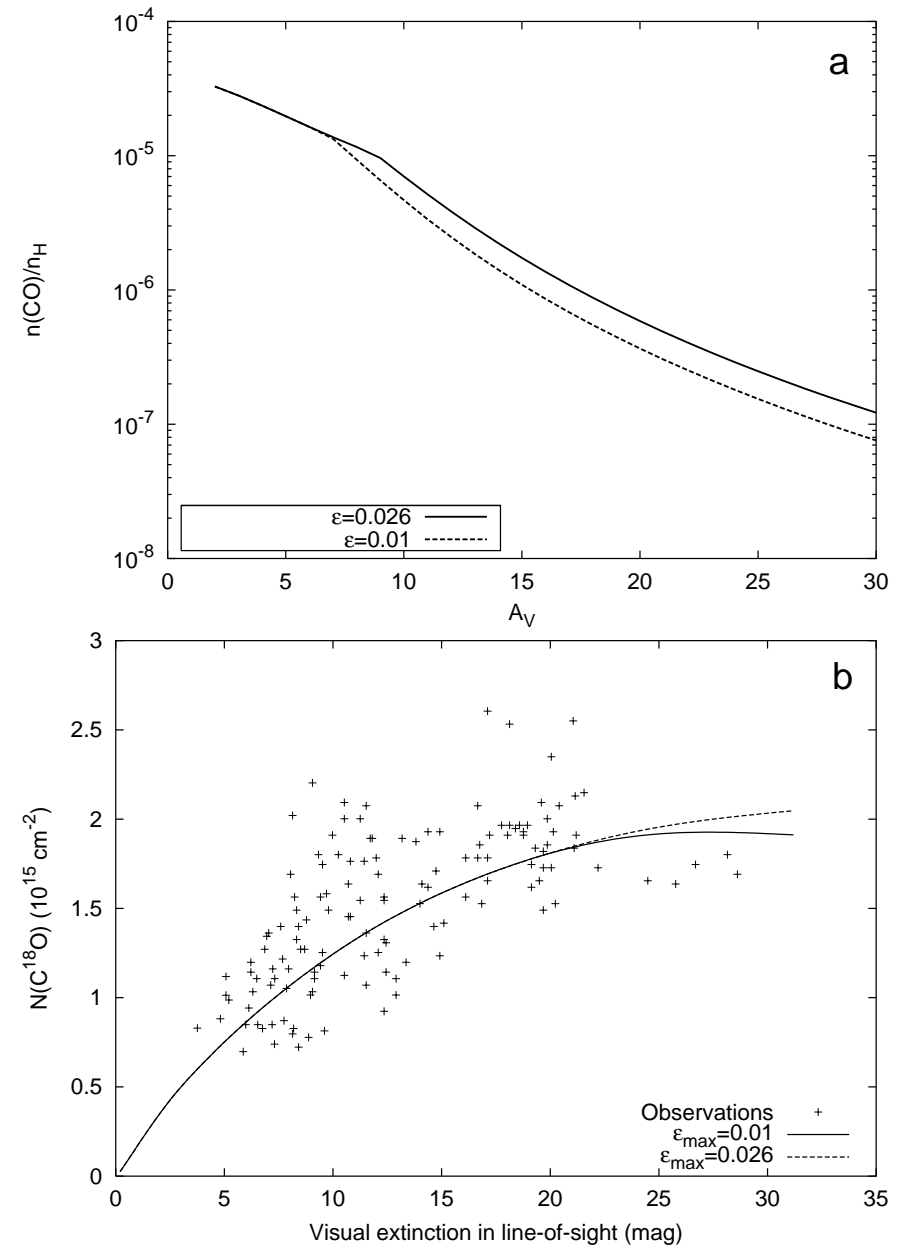

Fig. 12. The relative abundance of gaseous $\mathrm{CO}$ to total hydrogen a) and the $\mathrm{C}^{18} \mathrm{O}$ column density b) as a function of visual extinction along the line of sight for different maximum radical concentration $\varepsilon_{\max }$.

papers, the approximation $k_{\mathrm{crd}}(i)=f(70 \mathrm{~K}) k_{\text {evap }}(i, 70 \mathrm{~K})$ proposed by Hasegawa \& Herbst (1993) was used. Figure 9 shows the result obtained using this description of the whole grain heating. The approximation assumes that the sublimation of volatile species such as $\mathrm{CO}$ occurs near $70 \mathrm{~K}$, after passage of an energetic Fe nucleus through the $0.1 \mu \mathrm{m}$ grain. $f(70 \mathrm{~K})$ is the fraction of time spent by grains in the vicinity of $70 \mathrm{~K}$ and $k_{\text {evap }}(i, 70 \mathrm{~K})$ is the evaporation rate coefficient at $70 \mathrm{~K}$ for species $i$. However, in the dense core of the cloud, dust particles accrete ice mantles, which increase the size of the dust grain. The cosmic-ray particles can only heat a bigger grain with ice mantles to a lower temperature. For example, a relativistic $\mathrm{Fe}$ nucleus with energy $50 \mathrm{MeV}$ nucleon ${ }^{-1}$ can only heat dust particles of radius $0.14 \mu \mathrm{m}$ up to $40 \mathrm{~K}$. Because the sublimation rate depends exponentially on temperature, the approximation made by Hasegawa \& Herbst (1993) thus strongly overestimates the effect of the desorption due to cosmic-ray heating. Our treatment, using all the energy deposited by cosmic-ray particles and chemical energy release of radicals besides the energy to heat the grain to $26 \mathrm{~K}$ to sublimate $\mathrm{CO}$, is more reasonable and accurate, because the reasonable energy range of cosmic-ray spectra of several elemental nuclei, the size 

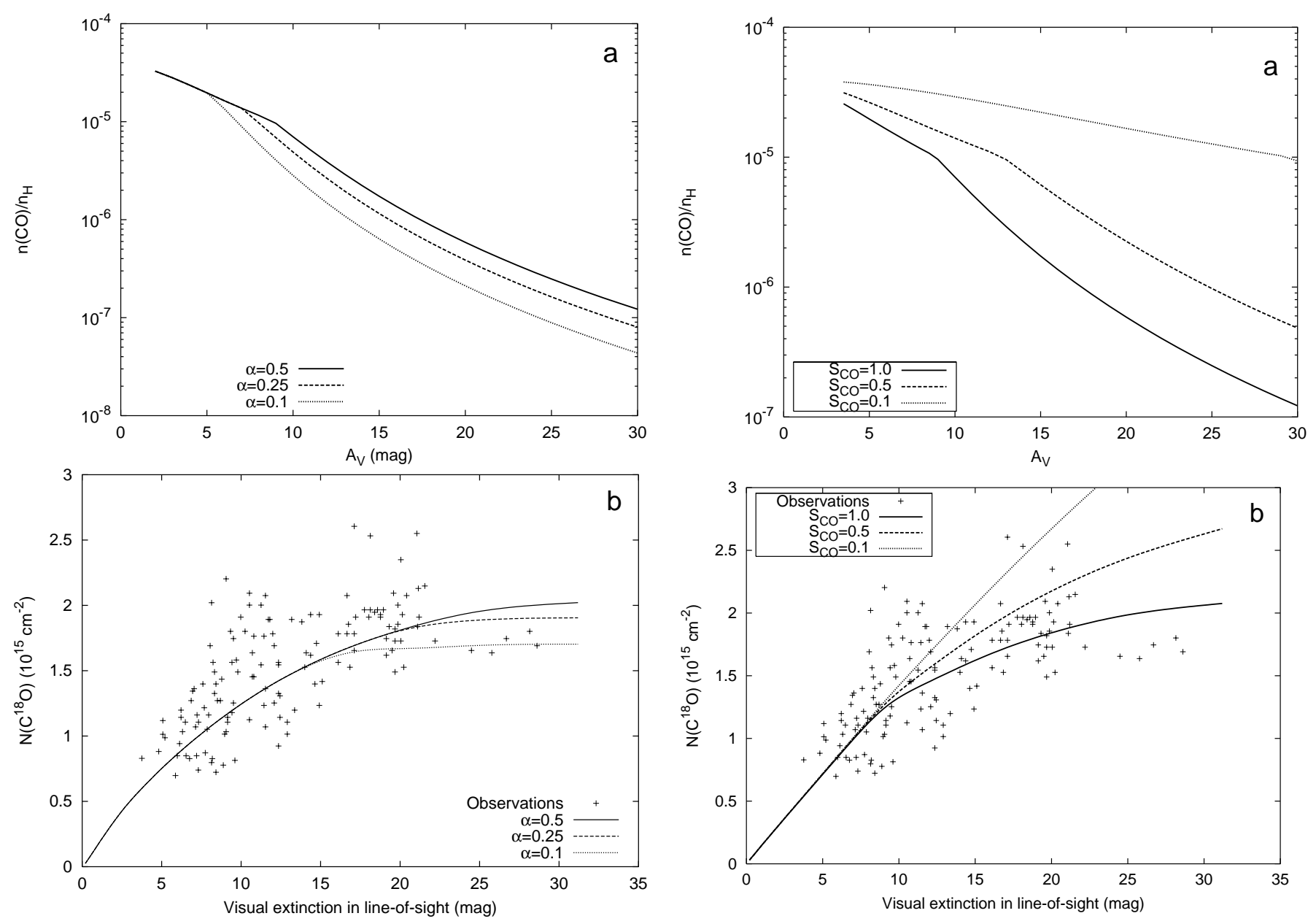

Fig. 13. The relative abundance of gaseous $\mathrm{CO}$ to total hydrogen a) and the $\mathrm{C}^{18} \mathrm{O}$ column density $\mathbf{b}$ ) as a function of visual extinction $A_{\mathrm{V}}$ for different values of the efficiency of free radical production by UV photons $\alpha$.

distribution of dust grains and the ice-mantle accretion are taken into account in calculating the desorption rates.

If the desorption due to the energy release of radicals produced by the cosmic-ray induced UV field is included, the observations can be fitted very well. The solid line in Fig. 9 is a calculation using all three desorption mechanisms. We conclude that the desorption due to the chemical energy release is the most important desorption mechanism in the core of a dense cloud. The cosmic-ray heating is only effective for smaller grains without ice mantle accretion.

An important result of our model is that the cosmic ray induced UV field is about 10 times more efficient in depositing energy in the ice than the direct cosmic ray energy deposition. This again emphasizes that the energy stored by UV photons in the form of free radicals in the ice is considerably larger than the energy deposited by cosmic-rays, and strengthens our result that chemical desorption is the most important desorption mechanism.

By varying the parameters, we find that variations in the maximum radical concentration $\varepsilon_{\max }$ and the free radical production by an ultraviolet photon $\alpha$ within reasonable limits do not change the model results very much. On the other hand,

Fig. 14. The relative abundance of gaseous $\mathrm{CO}$ to total hydrogen a) and the $\mathrm{C}^{18} \mathrm{O}$ column density $\mathbf{b}$ ) as a function of visual extinction $A_{\mathrm{V}}$ for different values of the sticking coefficients $S_{\mathrm{CO}}$ of CO.

the calculated CO column density is very sensitive to the cosmic-ray energy spectrum and thus the cosmic-ray ionization rate. Therefore some clues on the cosmic-ray ionization rate can be obtained by fitting the observation using this model.

Using the same parameters except the density profile, the model can fit the observations for both L977 and IC 5146. This supports the validity and robustness of our model. This model is by far the best fitting model to both sets of observations. Its application to future observations may therefore deepen our understanding of the nature of the balance between gases and ices in dense clouds.

Acknowledgements. We thank Dr. A. Li for providing the optical constants and some of the computer codes as well as fruitful discussions. We also acknowledge Dr. O. M. Shalabiea for helpful discussions. One of us (CS) wishes to thank the World Laboratory for a fellowship to perform this research.

\section{References}

Alves, J., Lada, C. J., \& Lada, E. A. 1999, ApJ, 515, 265

Alves, J., Lada, C. J., Lada, E. A., Kenyon, S. J., \& Phelps, R. 1998, ApJ, 506, 292

Al-Halabi, A., Fraser, H. J., van Dishoeck, E. F., \& Kroes, G. J. 2003, A\&A, submitted 
Bergin, E. A., Alves, J., Huard, T., \& Lada, C. J. 2002, ApJ, 570, L101 Bergin, E. A., Ciardi, D. R., Lada, C. J., Alves, J., \& Lada, E. A. 2001, ApJ, 557, 209

Bergin, E. A., Langer, W. D., \& Goldsmith, P. F. 1995, ApJ, 441, 222

Bloemen, H., Wijnands, R., Bennett, K., et al. 1994, A\&A, 281, L5

Bohlin, R. C., Savage, B. D., \& Drake, J. F. 1978, ApJ, 224, 132

Cecchi-Pestellini, C., \& Aiello, S. 1992, MNRAS, 258, 125

Chiar, J. E., Adamson, A. J., Kerr, T. H., \& Whittet, D. C. B. 1995, ApJ, 455, 234

Cowsik, R. 1980, ApJ, 241, 1195

Cravens, T. E., \& Dalgarno, A. 1978, ApJ, 219, 750

Cravens, T. E., Victor, G. A., \& Dalgarno, A. 1975, Planet. Space Sci., 23, 1059

d'Hendecourt, L. B., Allamandola, L. J., Baas, F., \& Greenberg, J. M. 1982, A\&A, 109, L12

d'Hendecourt, L. B., Allamandola, L. J., \& Greenberg, J. M. 1985, A\&A, 152, 130

Duley, W. W. \& Williams, D. A. 1993, MNRAS, 260, 37

Eichler, D. 1980, ApJ, 237, 809

Fano, U. 1963, Ann. Rev. Nucl. Sci., 13, 1

Flannery, B. P., Roberge, W. R., \& Rybicki, G. B. 1980, ApJ, 236, 598

Fransson, C., \& Epstein, R. I. 1980, ApJ, 242, 411

Greenberg, J. M. \& Yencha, A. J. 1973, in Interstellar Dust and Related Topics, IAU Symp., 52, 369

Grevesse, N., Noels, A., \& Sauval, A. J. 1996, in Cosmic Abundances, ASP Conf. Ser., 99, 117

Hasegawa, T. I. \& Herbst, E. 1993, MNRAS, 261, 83

Ip, W. H., \& Axford, W. I. 1985, A\&A, 149, 7

Kozlovsky, B., Ramaty, R., \& Lingenfelter, R. E. 1997, ApJ, 484, 286

Kramer, C., Alves, J., Lada, C. J., et al. 1999, A\&A, 342, 257

Lada, C. J., Alves, J. ., \& Lada, E. A. 1999, ApJ, 512, 250

Lada, C. J., Lada, E. A., Clemens, D. P., \& Bally, J. 1994, ApJ, 429, 694
Léger, A., Jura, M., \& Omont, A. 1985, A\&A, 144, 147

Li, A., \& Greenberg, J. M. 1997, A\&A, 323, 566

Mathis, J. S., Mezger, P. G., \& Panagia, N. 1983, A\&A, 128, 212

Meyer, J., O'C. Drury, L., \& Ellison, D. C. 1998, Space Sci. Rev., 86, 179

Okabe, H. 1978, Photochemistry of small molecules (New York, Wiley: Wiley-Interscience Publication)

Opal, C. B., Peterson, W. K., \& Beaty, E. C. 1971, J. Chem. Phys., 55, 4100

Pontoppidan, K. M., Fraser, H. J., Dartois, E., et al. 2003, A\&A, 408, 981

Prasad, S. S., \& Tarafdar, S. P. 1983, ApJ, 267, 603

Ramaty, R. 1996, A\&AS, 120, C373

Roberge, W. G. 1983, ApJ, 275, 292

Roberge, W. G. 1990, in Molecular Astrophysics, 288

Rudd, M. E., Kim, Y. K., Madison, D. H., \& Gallagher, J. W. 1985, Rev. Mod. Phys., 57, 965

Sandford, S. A., \& Allamandola, L. J. 1990, Icarus, 87, 188

Schutte, W. A. 1998, in Laboratory astrophysics and space research, ASSL, 236, 69

Schutte, W. A., \& Greenberg, J. M. 1991, A\&A, 244, 190

Simpson, J. A. 1983, Ann. Rev. Nucl. Part. Sci., 33, 323

Takahashi, J., \& Williams, D. A. 2000, MNRAS, 314, 273

Teixeira, T. C., \& Emerson, J. P. 1999, A\&A, 351, 292

Tielens, A. G. G. M., \& Hagen, W. 1982, A\&A, 114, 245

van der Tak, F. F. S., \& van Dishoeck, E. F. 2000, A\&A, 358, L79

Webber, W. R., \& Yushak, S. M. 1983, ApJ, 275, 391

Wiedenbeck, M. E., \& Greiner, D. E. 1980, ApJ, 239, L139

Willacy, K., \& Millar, T. J. 1998, MNRAS, 298, 562

Wilson, T. L., \& Rood, R. 1994, ARA\&A, 32, 191 